\title{
Approximating DEX Utility Functions with Methods UTA and ACUTA
}

\author{
Matej Mihelcic ${ }^{1,3}$, Marko Bohanec ${ }^{2}$ \\ ${ }^{1}$ Ruer Boškovi Institute, Division of Electronics, Laboratory for Information Systems, Croatia \\ 2 Jozef Stefan Institute, Department of Knowledge Technologies, Jamova 39, Ljubljana, Slovenia \\ matej.mihelcic@irb.hr \\ ${ }^{3}$ Jozef Stefan International Postgraduate School, Jamova 39, Ljubljana, Slovenia \\ marko.bohanec@ijs.si
}

\begin{abstract}
DEX is a qualitative multi-criteria decision analysis (MCDA) method, aimed at supporting decision makers in evaluating and choosing decision alternatives which has impact on security. We present results of a preliminary study in which we experimentally assessed the performance of two wellknown MCDA methods UTA and ACUTA to approximate qualitative DEX utility functions with piecewise-linear marginal utility functions. This is seen as a way to improve the sensitivity of qualitative models and provide a better insight in DEX utility functions. The results indicate that the approach is in principle feasible, but at this stage suffers from problems of convergence, insufficient sensitivity and inappropriate handling of symmetric functions.
\end{abstract}

Keywords: Security, Multi-criterial Decision Analysis

Received: 18 October 2018, Revised 19 December 2018, Accepted 7 January 2019

DOI: $10.6025 /$ isej/2019/6/1/1-8

(C) 2019 DLINE. All Rights Reserved

\section{Introduction}

Multi criteria decision analysis (MCDA) [1] is an approach concerned with structuring and solving decision problems involving multiple criteria. MCDA provides a number of methods [2] to create a decision model from information provided by the decision maker. This information can be given in many ways, for instance by constructing evaluation functions directly, by providing parameters (such as criteria weights) to some predefined functions, by giving examples of decisions, or by pairwise comparison of a subset of decision alternatives. Methods also differ in the representation of this information (e.g., quantitative or qualitative) and their primary aim (choosing the best alternative, ranking several alternatives, classifying alternatives into predefined discrete classes, etc.).

Bridging the gap between different MCDA methods is sometimes highly desirable and may have a great practical value. In this work, we try to combine two MCDA methods: DEX and UTA. DEX [3] is a qualitative method; it employs discrete attributes and discrete utility functions defined in a point-by-point way (see section 2.1). This makes DEX suitable for classifying decision

Information Security Education Journal Volume 6 Number 1 June 2019 
alternatives into discrete classes. On the other hand, UTA $[9,10]$ is a quantitative method that constructs numerical additive utility functions from a provided subset of alternatives (see section 2.2).

This work is motivated by the expectation that DEX's functionality would have been substantially enhanced if we were able to convert its discrete utility functions to numerical ones in some suitable way: first, the newly obtained numerical evaluations would facilitate an easy ranking and comparison of alternatives, especially those that are assigned the same class by DEX; second, the sheer form of numerical functions may tell us more about the properties of underlying DEX functions, which make them useful for verification, representation and justification of DEX models.

There have already been several attempts to approximate DEX utility functions with numeric ones for various purposes. A linear approximation method is commonly used in DEX to assess the importance (weights) of criteria [4,5]. An early method for ranking of alternatives and improving the sensitivity of evaluation has been proposed in [6] and is now referred to as QQ [7]. Recently, extensive research has been carried out to approximate DEX functions with copulas [7, 8]. However, no known attempts have been made so far to approximate DEX functions with piecewise-linear marginal utility functions, as provided by UTA.

The aim of this study was to experimentally assess the performance of UTA and its variant, ACUTA [11], on a collection of typical DEX functions. The experiments were carried out using two software tools: DEXi [4] to develop DEX functions and Decision Deck [12] to run (AC) UTA.

\section{Methods and Tools}

\subsection{DEX and DEXi}

DEX [3] is a qualitative MCDA method for the evaluation and analysis of decision alternatives, and is implemented in the software DEXi (http://kt.ijs.si/MarkoBohanec/dexi.html) [4]. In DEX, a decision model consists of hierarchically structured attributes: the hierarchy represents the decomposition of the decision problem into smaller sub problems, and attributes at higher levels of the hierarchy depend on those on lower levels. Figure 1 (left) shows an example of a tree of attributes for evaluating cars [4].

In the context of this paper, it is important to understand that all attributes in DEX models are qualitative and can take values represented by words; for instance, the attribute PRICE in Figure 1 can take the values high, medium and low. Furthermore, the aggregation of attributes at some level in the tree is defined by decision tables that consist of elementary decision rules. For example, the table in Figure 1 (right) defines the aggregation of two lower-level attributes PRICE and TECH.CHAR into the higher-level attribute CAR: the values of CAR are specified for all combinations of values of PRICE and TECH.CHAR. Essentially, this means that utility functions in DEX are discrete and defined in a point-by-point way. This is illustrated in Figure 2, which graphically represents the same function as in Figure 1, so that each row of Figure 1 is represented by a dot in Figure 2. The connecting lines are used only for visualization and are not part of function definition.

\begin{tabular}{|c|c|c|c|c|}
\hline \multirow{13}{*}{$\begin{array}{l}\square \text { CAR } \\
\square \text { PRICE } \\
\square \text { BUY.PRICE } \\
\square \text { MAINT.PRICE } \\
\square \text { TECH.CHAR. } \\
\square \text { COMFORT } \\
\square \leq \text { \#PERS } \\
\square \text { \#DOORS } \\
\square \text { SAFETY LUGGAGE }\end{array}$} & \multirow{2}{*}{\multicolumn{2}{|c|}{\begin{tabular}{l|l} 
& PRICE \\
1 & high \\
&
\end{tabular}}} & \multirow{2}{*}{\begin{tabular}{|l} 
TECH.CHAR, \\
bad
\end{tabular}} & \multirow{2}{*}{$\frac{\mid \text { CAR }}{\text { unaCC }}$} \\
\hline & & & & \\
\hline & & & acc & unacc \\
\hline & 3 & high & good & unacc \\
\hline & 4 & high & exc & unacc \\
\hline & 5 & medium & bad & unacc \\
\hline & 6 & medium & $a c c$ & aoc \\
\hline & 7 & medium & good & good \\
\hline & 8 & medium & exc & exc \\
\hline & 9 & low & bad & unacc \\
\hline & 10 & low & acc & good \\
\hline & 11 & low & good & exc \\
\hline & 12 & low & exc & exc \\
\hline
\end{tabular}

Figure 1. A DEX model and a utility function example [4] 


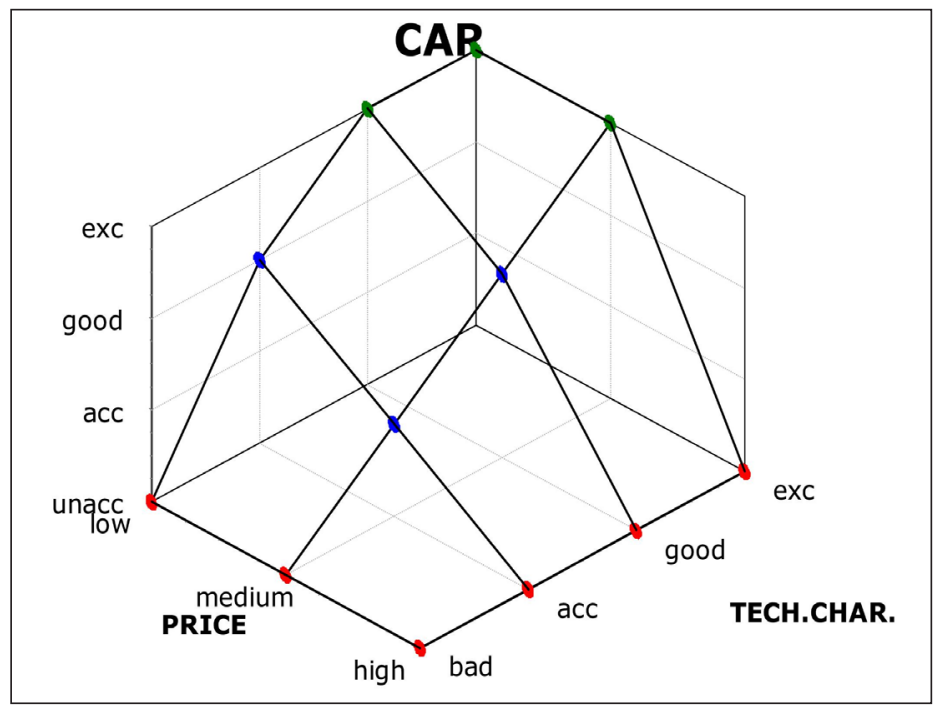

Figure 2. Graphical presentation of the CAR decision table

Formally, a DEX utility function is defined over a set of criteria $x_{1}, x_{2}, \ldots, x_{n}$ where all criteria are discrete and can take values from the corresponding value scales $D\left(x_{i}\right)$. A utility function $U$ maps $x$ to the higher-level attribute $y$ :

$$
U: D\left(x_{1}\right) \times D\left(x_{2}\right) \times \ldots \times D\left(x_{n}\right) \rightarrow D(y)
$$

$U$ is represented by a decision table that consists of elementary decision rules, where each rule defines the value of $U$ for some combination of argument values:

$$
\left\langle x_{1}, x_{2}, \ldots, x_{n}\right\rangle \rightarrow y
$$

For experiments in this study, we used a number of DEX utility functions, but in this paper we will present only three:

1. CAR function, as defined in Figures 1 and 2;

2. YM: defined over three attributes $(n=3)$, all the attributes have five values. The function is symmetric and represents a very common DEX function, which behaves as $\left(x_{1}, x_{2}, x_{3}\right)$ when any of the arguments takes the lowest possible value, and as a qualitative average of $x_{1}, x_{2}$ and $x_{3}$ otherwise.

3. YW: defined on the same space as YM, it represents an asymmetric DEX function defined with weights [4]; the weights assigned to the three arguments are $60 \%, 30 \%$ and $10 \%$, respectively.

All these functions are defined completely for all combinations of values of their arguments.

\subsection{UTAand ACUTA}

The UTA method (UTilité Additive) [9, 10] is used to assess utility functions which aggregate multiple criteria in a composite criterion used to rank the alternatives. Similarly as DEX, it uses a subjective ranking on a subset of the alternatives. On this basis, it creates piecewise-linear marginal utility functions.

For a set of alternatives $A, a \in A$, numerical criteria $g=\left(g_{1}, g_{2}, \ldots, g_{n}\right)$ and the utility function $U(g)=U\left(g_{1}, g_{2}, \ldots, g_{n}\right)$ the marginal utility functions $u_{i}$ are approximated with:

$$
u_{i}\left[g_{i}(a)\right]=u_{i}\left(g_{i}^{J}\right)+\frac{g_{i}(a)-g_{i}^{J}}{g_{i}^{J+1}-g_{i}^{J}}\left[u_{i}\left(g_{i}^{J+I}\right)-u_{i}\left(g_{i}^{J}\right)\right]
$$


It is assumed that each attribute's values are divided to $\alpha_{i}-1$ equally-sized intervals $\left[g_{i}^{J}, g_{i}^{J+1}\right]$

The marginal utility functions $u_{i}$ are constructed by solving the linear programming problem

$$
\min F=\Sigma_{a \in A} \sigma(a)
$$

under the constraints:

$$
\begin{aligned}
& \sum_{i=1}^{n} u_{i}\left[g_{i}(a)\right]-u_{i}\left[g_{i}(b)\right]+\sigma(a)-\sigma(b) \geq \delta, a P b \\
& \sum_{i=1}^{n} u_{i}\left[g_{i}(a)\right]-u_{i}\left[g_{i}(b)\right]+\sigma(a)-\sigma(b)=0, a I b \\
& u_{i}\left(g_{i}^{J+1}\right)-u_{i}\left(g_{i}^{J}\right) \geq s_{i}, \forall i \in\{1, \ldots, n\}, J \in\{1, \ldots, \alpha\} \\
& \sum_{i=1}^{n} u_{i}\left(g_{i}^{*}\right)=1 \\
& u_{i}\left(g_{i^{*}}\right)=0, u_{i}\left(g_{i}^{J}\right) \geq 0, \sigma(a) \geq 0, \\
& \forall i \in\{1, \ldots, n\}, J \in\{1, \ldots, \alpha\} \forall a \in A
\end{aligned}
$$

Here, $\sigma(a)$ denotes potential error relative to the starting utility $U[g(a)] . g_{i}{ }^{*}$ and $g_{i^{*}}$ denote the high and low bounds of $g_{i}$ respectively. $P$ and $I$ respectively denote strict preference and indifference relations.

In some cases there can be many utility functions that can represent the preferences specified. The utility functions are then assessed by means of post-optimality analysis [9].

The ACUTA method [11] offers an improvement upon UTA. It proceeds by finding an analytic center of the additive value functions that are compatible with some user assessments of preferences. In this way, ACUTA solves the model selection problem present in the UTA method when there are multiple valid solutions. Similarly as UTA, it constructs marginal utility functions by solving a constrained optimization problem, see [11] for details.

In order to approximate DEX utility functions with (AC)UTA, we mapped qualitative DEX attributes $x \in D(x)$ to equidistant numerical scales $g=[1,|D(x)|]$.

\subsection{Decision Deck and Diviz}

The Decision Deck (http://www.decision-deck.org/project/) is a project aimed at developing an open-source MCDA software platform [12]. Diviz is a software component developed in Decision Deck aimed at designing, executing and sharing MCDA methods, algorithms and experiments [12]. Diviz enables combining programs that implement MCDA algorithms in a modular way and connecting them in terms of workflows.

Figure 3 shows the workflow used in this study to run ACUTA. The input consists of six datasets. The Criteria file contains names and ID's of the decision criteria, the Alternatives file contains names and ID's of the alternatives, the PerformanceTable file contains the attribute values for each alternative, the AlternativeValues file contains a ranking of a small sample of the alternatives determined by the decision maker (usually called a priori ranking), the PreferenceDirection file indicates preferred optimization direction, and the NumberofSegments file defines the number of segments to which the attribute values are split. The output of the workflow is a rank of alternatives given their attribute values and the a priori ranking.

\section{Results}

Several problems were detected when we attempted to approximate DEXi utility functions with (AC)UTA in Diviz. First, the standard UTA method could not handle DEX utility functions and returned an error message:

4 Information Security Education Journal Volume 6 Number 1 June 2019




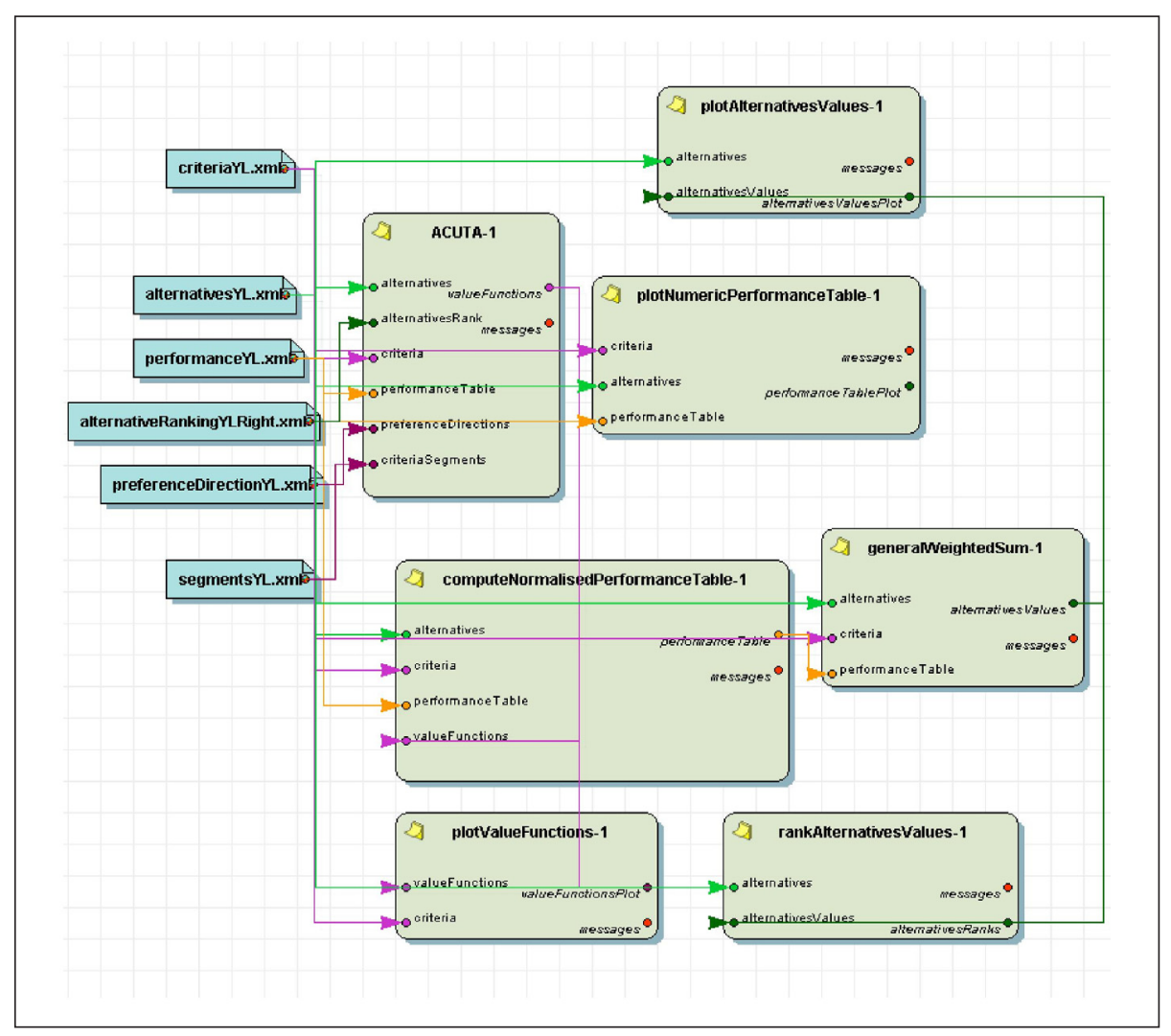

Figure 3. The ACUTA decision support workflow

\section{Execution terminated, but no result were produced: \\ you probably hit a bug in the service. [...]}

In order to get any results, we had to take only a subset of the rules, that is, remove a subset of entries from the UTA performance table.

The second problem with UTA was setting the a priori alternative ranking (i.e., the target attribute) in a way that would allow the method to converge. We used the inverse DEX attribute label score as a priori rank for the UTA method. As a result, all the alternatives with the same DEXi label score were indifferent for UTA. This required us to take only a small, targeted subset of available a priori ranks.

Overall, the results produced by UTA were poor and did not accurately approximate input functions.

The ACUTA method performed much better and the models were built on the whole domain of the DEX utility functions. However, we did experience convergence issues when using inverse DEX label attribute score as a priori rank for all the alternatives, so we had to take a subset that allowed the method to converge. The convergence error message reported by ACUTA was as follows:

Error - failed to converge, due to bad information. Please check your data, rescale the problem, or try with less constraints.

The results for the Car utility function are shown in Figure 4, where $g 1$ and $g 2$ indicate DEX attributes PRICE and TECH.CHAR. Both marginal utility functions properly increase, and in $g 1$ the relations between utility values in points 1 , and 2 appear right, 


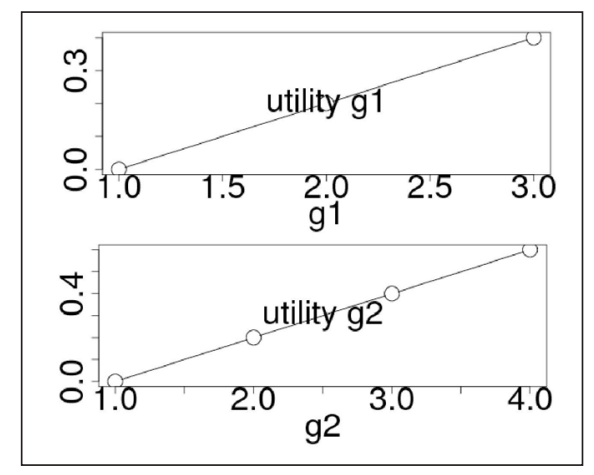

Figure 4. ACUTA results for DEX function Car

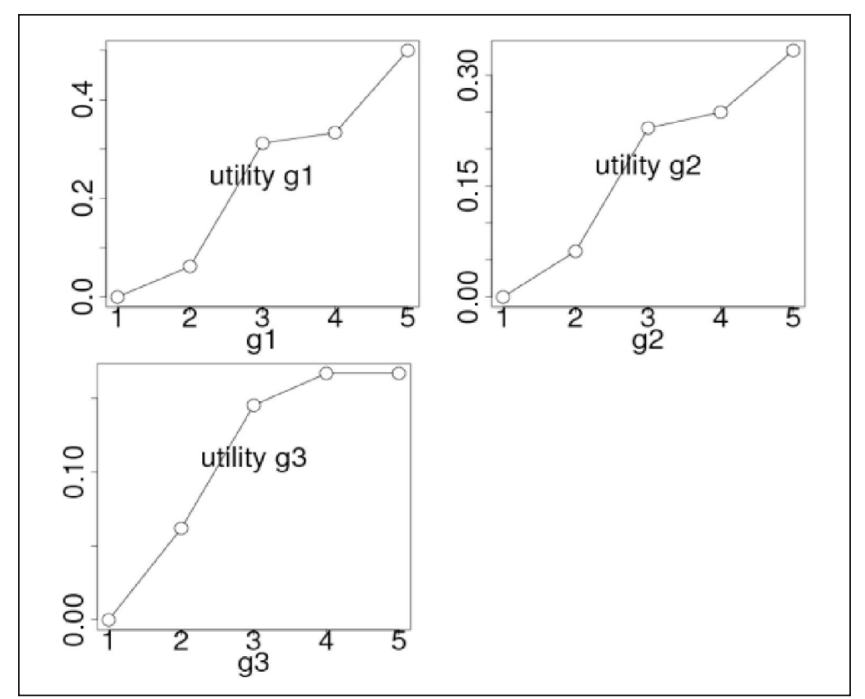

Figure 5. ACUTA results for DEX function YM

however utility value in point 3 is too high. We noticed similar behavior in function $g 2$.

Figure 5 shows results for the DEX function YM. In our opinion, marginal utility functions approximate YM quite well, however they indicate a common problem encountered in the experiments: YM is symmetric, therefore ACUTA's marginal functions should be equal to each other, but they are not. In this way, the resulting representation does not properly capture the symmetricity of the original function.

Marginal utility functions in Figure 6 correctly indicate that $\mathrm{YW}$ is asymmetric and, observing function's maximum values, that the attributes $g 1, g 2$, and $g 3$ are less and less important. However, some sections of these functions are almost constant, which does not hold in the original function.

\section{Conclusion}

In this preliminary study we tried to approximate several DEX utility functions by using the basic UTA method and its derivative, ACUTA. In general, the approach turned out to be feasible, producing marginal utility functions from DEX utility functions, which are defined by points in a discrete multidimensional space. The obtained functions are easy to interpret and do provide useful information about DEX attributes and scales (e.g., numeric utility value for each discrete attribute value), and the underlying DEX utility functions (e.g., about relative importance of attributes). Therefore, the approach is useful for representing and understanding DEX utility functions: the representation consists of a set of additive utility functions that represent attribute trends and importance's that cannot be easily observed by examining DEX utility functions themselves.

6 Information Security Education Journal Volume 6 Number 1 June 2019




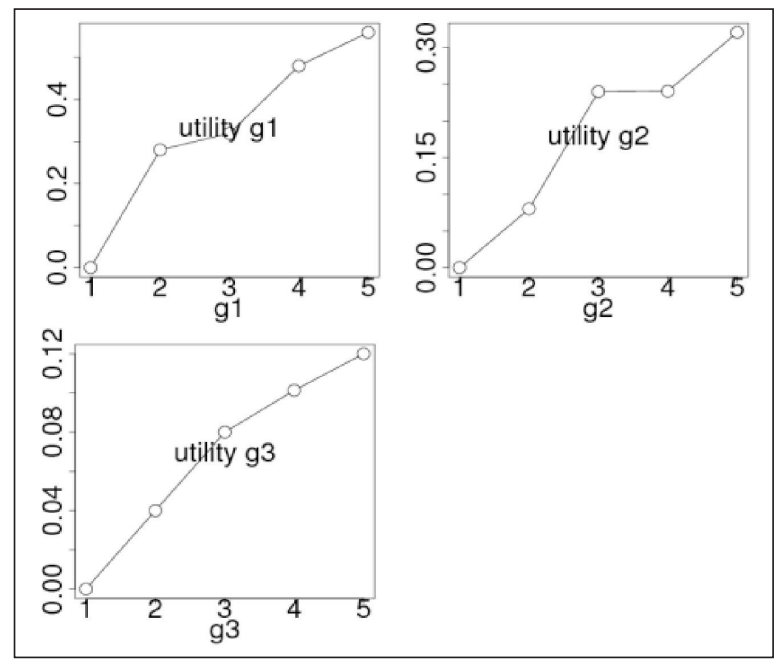

Figure 6. ACUTA results for DEX function YW

On the other hand, we encountered several problems with the methods and their implementation. UTA rarely gives any results on the original DEX functions, and even after tweaking the inputs the results were unsatisfactory. ACUTA performs much better, it can work on the whole domain of the DEX function, but the a priori rank subset needs to be carefully chosen in order to avoid convergence problems. The theoretical reasons for convergence problems of these methods are still to be determined.

Marginal utility functions, generated by ACUTA, in principle appropriately represent the marginal behavior of DEX attributes, but they exhibit two common problems:

- Insufficient sensitivity to changes of attribute values (some sections of ACUTA functions are (almost) constant even though the underlying DEX function is not);

- Inappropriately representing symmetric DEX functions with mutually different marginal utility functions.

In future work, we wish to theoretically and empirically address these issues and alleviate these problems, either by adopting some other method from the rich set of UTA related methods [10], by adapting (AC)UTA to specific properties of DEX functions, or by developing entirely new methods. Eventually, the method should be able to deal with all type of DEX functions, including large ones, incompletely defined ones and those defined with distributions of classes.

\section{References}

[1] Ehrgott, M., Figueira, J. R., Greco, S. (2010). Trends in Multiple Criteria Decision Analysis, International Series in Operations Research \& Management Science, Vol. 142, New York: Springer.

[2] Figueira, J. R., Greco, S., Ehrgott, M. (2005). Multiple Criteria Decision Analysis: State of the Art Surveys, Boston: Springer.

[3] Bohanec, M., Rajkovi, V., Bratko, I., Zupan, B., Znidarši, M. (2013). DEX methodology: Three decades of qualitative multiattribute modelling. Informatica 37, 49-54.

[4] Bohanec, M. (2013). DEXi: Program for Multi-Attribute Decision Making, User's Manual, Version 4.00. IJS Report DP11340, Ljubljana: Jo•ef Stefan Institute.

[5] Bohanec, M., Zupan, B. (2004). A function-decomposition method for development of hierarchical multi-attribute decision models. Decision Support Systems 36, 215-233.

[6] Bohanec, M., Urh, B., Rajkovi, V. (1992). Evaluating options by combined qualitative and quantitative methods. Acta Psychologica 80, 67-89.

[7] Mileva-Boshkoska B., Bohanec, M. (2012). A method for ranking non-linear qualitative decision preferences using copulas. 
International Journal of Decision Support System Technology 4 (2) 42-58.

[8] Mileva-Boshkoska, B., Bohanec, M., Boškoski, P., Juricic, D. (2013). Copula-based decision support system for quality ranking in the manufacturing of electronically commutated motors. Journal of Intelligent Manufacturing, doi: 10.1007/s10845013-0781-7.

[9] Jacquet-Lagreze, E., Siskos, J. (1982). Assessing a set of additive utility functions for multicriteria decisionmaking, the UTA method, European Journal of Operational Research, 10 (2) 151-164.

[10] Siskos, Y., Grigoroudis, E., Matsatsinis, N. F. (2005). UTA methods. In: Multiple Criteria Decision Analysis: State of the Art Surveys, 297-343, Boston: Springer.

[11] Bous, G., Fortemps, P., Glineur, F., Pirlot, M. (2010). ACUTA: A novel method for eliciting additive value functions on the basis of holistic preference statements, European Journal of Operational Research, 206 (2) 435-444.

[12] Ros, J. C. (2011). Introduction to Decision Deck-Diviz: Examples and User Guide, Technical report DEIM-RT- 11-001, Tarragona: Universitat Rovira i Virgili. 